\title{
Artigo/Article
}

\section{Estudo clínico-epidemiológico da doença de Chagas no distrito de Serra Azul, Mateus Leme, centro-oeste do Estado de Minas Gerais}

\author{
Clinic and epidemiological study on Chagas disease in the Serra Azul district of Mateus Leme, \\ central-western region of the State of Minas Gerais, Brazil
}

\author{
Élvio Marques da Silva ${ }^{1,2,3}$, Manoel Otávio da Costa Rocha ${ }^{1}$, Rian César Silva ${ }^{2}$, Gilmar do Carmo Paixão ${ }^{2}$, \\ Haendel Buzzati ${ }^{2}$, Alessandra Nogueira Santos ${ }^{3}$ e Maria do Carmo Pereira Nunes ${ }^{1}$
}

\begin{abstract}
RESUMO
Introdução: A doença de Chagas permanece como grave problema de saúde pública na América Latina, mesmo com o controle de sua transmissão. Esse estudo avaliou a prevalência da doença de Chagas no distrito de Serra Azul, Centro-Oeste de Minas Gerais, identificando as anormalidades eletrocardiografias dos indivíduos com sorologia positiva. Métodos: A sorologia para doença de Chagas foi realizada em 676 indivíduos ( $48 \%$ da população). O exame clínico e o eletrocardiograma (ECG) foram realizados na população sorologicamente positiva. Selecionou-se um grupo controle para comparação dos dados do ECG. Resultados: A população estudada residia principalmente em área rural, com baixo nível sóciocultural. Quatorze indivíduos foram positivos, com prevalência de 2,1\%. Os indivíduos chagásicos eram de faixa etária mais avançada ( $67 v s 39$ anos; $\mathrm{p}<0,001)$. As anormalidades ao ECG nos chagásicos foram frequentes (79\%). O ECG dos soropositivos mostrou maior prevalência de extrassístoles ventriculares, distúrbio da condução do ramo direito isolado ou associado ao bloqueio da divisão ântero-superior em relação ao grupo controle. Conclusões: A prevalência da doença de Chagas no distrito de Serra Azul foi de 2,1\%. Os soropositivos estavam em faixa etária mais elevada, sugerindo controle da transmissão. As anormalidades eletrocardiográficas foram frequentes, predominando distúrbio de condução do ramo direito.
\end{abstract}

Palavras-chaves: Doença de Chagas. Sorologia para Trypanosoma cruzi. Prevalência. Eletrocardiograma.

\begin{abstract}
Introduction: Chagas disease remains a severe public health problem in Latin America, despite control over its transmission. This study evaluated the prevalence of Chagas disease in the Serra Azul district, in the central-western region of the State of Minas Gerais, through identifying electrocardiographic (EKG) abnormalities among serologically positive individuals. Methods: Specific serological tests for Chagas disease were carried out on 676 individuals (48\% of the population). Clinical examinations and EKGs were performed on the serologically positive population. A control group was selected for comparison with the EKG data. Results: The study population lived mainly in rural areas, with low sociocultural level. Forty subjects were positive (prevalence of $2.1 \%$ ). The individuals with Chagas disease were in an older age group (67 vs. 39 years; $\mathrm{p}<0.001$ ). EKG abnormalities were frequent among the individuals with Chagas disease (79\%). Among the seropositive individuals, the EKGs showed greater prevalence of ventricular premature beats and conduction disorder of the right branch alone or in association with anterosuperior division blockage, in relation to the controls. Conclusions: The prevalence of Chagas disease in the Serra Azul district was $2.1 \%$. The seropositive individuals were in an older group, thus suggesting that transmission had been brought under control. EKG abnormalities occurred frequently, and conduction disorders of the right branch predominated.
\end{abstract}

Key-words: Chagas disease. Serological test for Trypanosoma cruzi. Prevalence. Electrocardiogram.

1. Pós-graduação em Ciências da Saúde: Infectologia e Medicina Tropical, Faculdade de Medicina da Universidade Federal de Minas Gerais, Belo Horizonte, MG. 2. Faculdade de Farmácia, Universidade de Itaúna, Itaúna, MG. 3. Casa de Caridade Manoel Gonçalves, Itaúna, MG.

Endereço para correspondência : Dr. Élvio Marques da Silva. Rua Diógenes Nogueira 11/sala 8, Edifício Central Park, Centro, 35680-040 Itaúna, MG.

Telefax: 37 3242-3585

e-mail: emarques@cardiol.br

Recebido para publicação em 26/05/2009

Aceito em 09/01/2010

\section{INTRODUÇÃO}

A doença de Chagas constitui um dos principais problemas médico-sociais brasileiros e um grave problema de saúde pública na América Latina. Estimativa atual da Organização Mundial de Saúde (OMS) indica prevalência de infecção pelo Trypanosoma cruzi em torno de 13 milhões de pessoas em 15 países, com incidência anual de 200.000 casos $^{1-3}$. No Brasil, com o controle das transmissões vetorial e transfusional, estima-se em dois a três milhões de pessoas infectadas, com prevalência atual da infecção inferior a $0,2 \%{ }^{4}$. Minas Gerais é considerado um dos estados brasileiros com maior prevalência da endemia chagásica. A região centro-oeste sempre apresentou elevada endemicidade, destacando-se o município de Bambuí, onde as ações de controle da transmissão vetorial tiveram início na década de $40^{5}$. Análises recentes da região centro-oeste de Minas Gerais têm demonstrado quatro espécies de triatomíneos invadindo domicílios (Panstrongylus megistus, Panstrongylus diasi, Rhodnius neglectus, Triatoma sordida), com um índice de infecção de 1,3\% .

Assim, a motivação do presente estudo se deveu à observação de que no centro-oeste de Minas Gerais, em cidades como Mateus Leme, Itaúna, Itatiaiuçu, Itaguara, dentre outras, era grande o número de pacientes que se apresentavam nas Unidades de Saúde, Ambulatórios Médicos e Serviços de Urgência com arritmias cardíacas, insuficiência cardíaca, e morte. Especialmente, no povoado de Serra Azul, distrito de Mateus Leme, observações médicas isoladas sugeriam ser grande o número de pessoas infectadas pelo Trypanosoma cruzi e muitas não tinham o diagnóstico da doença.

\section{MÉTODOS}

O município de Mateus Leme situa-se na região metropolitana de Belo Horizonte, possui uma área de $302,589 \mathrm{~km}^{2}$ e o distrito ou povoado de Serra Azul que pertence a Mateus Leme, está a $60 \mathrm{~km}$ da capital 
mineira. De acordo com dados da Secretaria Municipal de Saúde, do Sistema de Informação de Atenção Básica, através famílias cadastradas de 2007-Modelo Programa Saúde da Família, total de 1.405 pessoas está registrado em Serra Azul. A economia do distrito é baseada quase exclusivamente na agricultura através de hortifruticultura que abastece os armazéns da Central Ceasa em Belo Horizonte.

Considerou-se para o cálculo do tamanho da amostra populacional a prevalência de doença de Chagas no Estado de Minas Gerais de $8,8 \%$ e no país com uma variação de prevalência de 1,5 a $12,5 \%{ }^{6}$ e com base nos seguintes parâmetros: $\alpha$ - bicaudal $=0,05$ (precisão absoluta com $5 \%$ para cada lado da estimativa) e com a média de variação da prevalência de 3 a 7\%, encontrando-se 457 indivíduos.

A população total foi convidada a realizar a triagem sorológica no período de maio a agosto de 2007 . Todo o povoado foi visitado, extraindo-se informações demográficas e socioeconômicas.

O estudo foi realizado em duas etapas, no distrito de Serra Azul pertencente à cidade de Mateus Leme, Centro-Oeste do Estado de Minas Gerais, Brasil. A primeira consistiu na realização de exames sorológicos e um questionário, na residência das pessoas ou no comércio. Na segunda etapa, os indivíduos com sorologia positiva foram submetidos ao exame clínico e eletrocardiograma de repouso de 12 derivações, no Posto de Saúde do povoado de Serra Azul.

Selecionou-se, também, um grupo controle constituído por indivíduos com sorologia negativa, na proporção de $2: 1$, com características demográficas semelhantes aos soropositivos.

Foram realizados testes sorológicos de hemaglutinação indireta e ELISA por bioquímicos do Laboratório Carlos Chagas, em Itaúna-MG.

$\mathrm{Na}$ população sorologicamente positiva, foi realizado o exame clínico e eletrocardiograma de repouso. $\mathrm{O}$ exame clínico na população soropositiva consistiu em anamnese e exame físico dirigidos para o aparelho cardiovascular, especialmente avaliação dos pulsos, pressão arterial sistêmica e ausculta cardíaca durante dois minutos à procura de distúrbios do ritmo cardíaco. Realizou-se eletrocardiograma convencional de repouso utilizando-se as 12 derivações num total de 14 indivíduos soropositivos e em 30 pacientes com sorologia negativa, o grupo controle. Foi analisado o ritmo e calculada a frequência cardíaca.

Foram analisadas as variáveis qualitativas pela estatística descritiva, sendo elaboradas as distribuições de frequência e descrições de achados demográficos. A média de idade dos participantes com sorologia positiva foi comparada à idade dos demais, através do teste ' $\mathfrak{t}$ ' de Student. A distribuição por sexo foi comparada através do teste de qui-quadrado. Em todos os testes, utilizou-se a probabilidade de significância (p) menor que 0,05 como necessários para rejeição da hipótese nula.

\section{Ética}

O Comitê de Ética em Pesquisa da UFMG, (COEP/UFMG) aprovou este projeto de pesquisa em 28 de junho de $2007 \mathrm{com}$ parecer número ETIC 168/07 e os participantes foram esclarecidos a respeito do protocolo de estudo, tendo todos eles concordado na sua participação e assinado o termo de consentimento.

\section{RESULTADOS}

Foram estudados 676 indivíduos, com idade média foram $39,6 \pm 18$ anos, variando de 2 a 97 anos. Os participantes foram distribuídos de forma homogênea em relação ao sexo, sendo 380 (56\%) femininos e 296 (44\%) masculinos. A Tabela 1 mostra a faixa etária da população total do povoado em comparação à população estudada.

De 0 aos 14 anos, num total de 343 crianças e adolescentes, 62 foram estudadas. Em relação ao total de 676 indivíduos, submetidos aos exames sorológicos, somente 2,2\% tinham entre 0 e 6 anos e $7 \%$ entre 7 e 14 anos.

TABELA 1 - Dados da população total do distrito de Serra Azul pelo Programa Saúde da Família, comparando-se com a população estudada, conforme a faixa etária.

\begin{tabular}{lcccc}
\hline $\begin{array}{l}\text { Idade } \\
\text { (anos) }\end{array}$ & $\begin{array}{c}\text { População } \\
\text { do distrito* }\end{array}$ & $\begin{array}{c}\text { População } \\
\text { estudada** }\end{array}$ & $\begin{array}{c}\text { Porcentagem } \\
(\%)\end{array}$ & $\begin{array}{c}\text { Pessoas } \\
\text { submetidas ao } \\
\text { exame sorológico** } \\
(\%)\end{array}$ \\
\hline $0-6$ & 149 & 15 & 10,0 & 2,2 \\
$7-14$ & 194 & 47 & 24,0 & 7,0 \\
$15-39$ & 573 & 285 & 49,0 & 42,1 \\
$40-59$ & 332 & 221 & 66,0 & 32,7 \\
$>60$ & 157 & 108 & 68,0 & 16,0 \\
\hline
\end{tabular}

${ }^{*} \mathrm{n}^{\mathrm{o}}=1405,{ }^{* *} \mathrm{n}^{\mathrm{o}}=676$.

A sorologia para Trypanosoma cruzi foi positiva em 15 indivíduos. Um caso foi excluído por não pertencer ao distrito de Serra Azul. A prevalência foi de $2 \%$ (14 pacientes) da amostra da população estudada (675 indivíduos).

Em relação ao sexo, observou-se que dez (72\%) eram mulheres e quatro (28\%) homens, sem diferença em relação à população total $(\mathrm{p}=0,49)$. Observou-se que os pacientes com sorologia positiva prevaleceram na população idosa (67 vs 39 anos; $\mathrm{p}<0,001$ ) (Figura 1). Houve aparecimento de casos com sorologia positiva a partir dos 51 anos e idade máxima de 89 anos, com média do grupo de 67 anos.

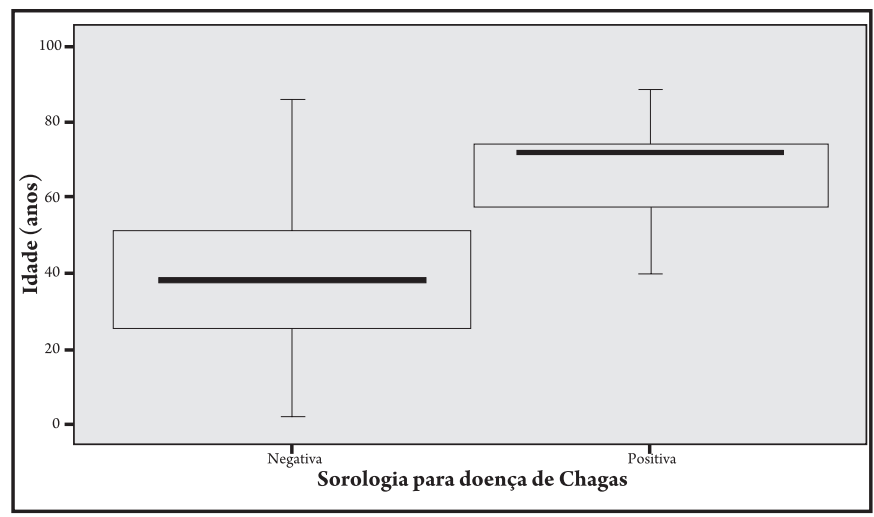

FIGURA 1 - Distribuição dos 675 pacientes de acordo com a sorologia para doença de Chagas, em relação à idade, no distrito de Serra Azul, Mateus Leme, Minas Gerais.

Dos sorológicos positivos, todos residiam na zona rural, com predomínio de brancos (72\%). Em relação à profissão, a maioria (78\%) eram lavradores e conforme os graus de escolaridade, 13 (93\%) tinham o ensino fundamental incompleto. Em relação à epidemiologia prévia, apenas um indivíduo com sorologia positiva relatou ter sido submetido à transfusão de sangue no Hospital de Mateus Leme, há trinta anos.

Dois (14\%) referiram contato com o inseto vetor, nove (64\%) não sabiam e dois (14\%) negaram o contato. A presença de sorologia positiva para Trypanosoma cruzi era desconhecida para 8 (57\%) indivíduos e $6(43 \%)$ conheciam a sua doença. Na segunda etapa do trabalho, todos os 14 indivíduos com sorologia positiva e os 
TABELA 2 - Alterações eletrocardiográficas conforme o método de Buenos Aires em chagásicos e controles, residentes no povoado de Serra Azul, Mateus Leme, Minas Gerais.

\begin{tabular}{|c|c|c|c|c|c|}
\hline \multirow[b]{2}{*}{ Eletrocardiograma } & \multicolumn{2}{|c|}{$\begin{array}{c}\text { Chagásicos } \\
\left(\mathrm{n}^{\mathrm{o}}=14\right)\end{array}$} & \multicolumn{2}{|c|}{$\begin{array}{l}\text { Controle } \\
\left(\mathrm{n}^{\mathrm{o}}=30\right)\end{array}$} & \multirow[b]{2}{*}{$\mathrm{p}$} \\
\hline & $\overline{\mathrm{n}} \mathrm{o}$ & $\%$ & $\mathrm{n}^{\mathrm{o}}$ & $\%$ & \\
\hline Normal & 3 & 21,0 & 12 & 40,0 & \\
\hline Com alterações & 11 & 79,0 & 18 & 60,0 & 0,226 \\
\hline \multicolumn{6}{|l|}{ Tipos de alterações } \\
\hline bradicardia sinusal & 02 & 14,0 & 04 & 13,0 & 0,932 \\
\hline fibrilação atrial & 1 & 7,0 & - & - & - \\
\hline extrassístoles supraventriculares & - & - & - & - & - \\
\hline extrassístoles ventriculares & 5 & 36,0 & 1 & 3,0 & 0,004 \\
\hline bloqueio átrio-ventricular de primeiro grau & 1 & 7,0 & 2 & 7,0 & 0,953 \\
\hline bloqueio de grau avançado do ramo direito & 8 & 57,0 & 2 & 7,0 & $<0,001$ \\
\hline bloqueio de grau não avançado do ramo direito & - & - & - & - & - \\
\hline bloqueio da divisão ântero-superior & 1 & 7,0 & 3 & 10,0 & 0,759 \\
\hline bloqueio de grau avançado do ramo direito associado a bloqueio da divisão ântero-superior & 4 & 29,0 & 1 & 3,0 & 0,014 \\
\hline bloqueio de grau avançado do ramo esquerdo & - & - & - & - & - \\
\hline hipertrofia ventricular esquerda & - & - & & 1,0 & 3 \\
\hline hipertrofia ventricular direita & - & - & & - & - \\
\hline alteração do segmento $\mathrm{ST}$ e $\mathrm{T}$ & 4 & 29,0 & 11 & 37,0 & 0,598 \\
\hline
\end{tabular}

30 controles foram submetidos, no Posto de Saúde da Secretaria Municipal de Mateus Leme, ao exame clínico, com ênfase ao aparelho cardiovascular.

O sintoma mais frequente foi dispnéia aos esforços, referida por $10(71 \%)$ pessoas e destes, quatro também relataram edema. Dos 14 soropositivos, 5 (36\%), referiram constipação intestinal superior a 5 dias e não houve menção de disfagia.

Em relação à ausculta cardíaca durante 2 minutos, em 11 (79\%) dos estudados foi normal e em três (21\%) detectou-se distúrbio do ritmo. A frequência cardíaca média foi de $75 \mathrm{bpm}$ e a pressão arterial de 120 x $75 \mathrm{mmHg}$. Os medicamentos mais usados foram: captopril, enalapril, hidroclorotiazida, AAS e amiodarona.

O eletrocardiograma foi considerado anormal em 11 (79\%) dos soropositivos (Tabela 2). As alterações mais frequentes foram os bloqueios completo do ramo direito isolado ou associado ao bloqueio da divisão ântero-superior do ramo esquerdo, detectadas em $57 \%$ dos casos.

Outras alterações eletrocardiográficas comuns na doença de Chagas, como extrassistolia ventricular e bradicardia sinusal foram verificadas, respectivamente, em cinco (35\%) e dois (14\%) pacientes. Considerando-se apenas o exame clínico e eletrocardiograma para classificar a forma clínica de apresentação da doença de Chagas nos pacientes com sorologia positiva, verificou-se ausência de cardiopatia em três (21\%). Os demais (79\%) participantes apresentavam alguma anormalidade ao exame clínico e/ou eletrocardiograma, com distúrbio de condução do ramo direito, típico da cardiopatia chagásica, detectado em oito (57\%) indivíduos.

Levando-se em conta a idade dos pacientes chagásicos, verificouse concomitantemente, outras co-morbidades como hipertensão arterial (64\%) e diabetes mellitus (14\%). Apenas um dos pacientes apresentou insuficiência cardíaca descompensada secundário à miocardiopatia chagásica, com várias internações, evoluindo para o óbito durante o estudo de campo. Todos os participantes do grupo controle submeteram-se ao exame clínico e ao eletrocardiograma. A idade média foi de $63 \pm 10$ anos, sem diferença em relação a grupo estudo ( $<<0,444)$, com 13 (43\%) homens e 17 (57\%) mulheres, também semelhantes ao grupo estudo $(\mathrm{p}<0,349)$.

\section{DISCUSSÃO}

O controle atual da transmissão da doença de Chagas resultou em queda acentuada da incidência de novos casos nos últimos anos. Isto não significa, entretanto, o fim das repercussões de uma doença crônica com elevado impacto sócio-econômico. Para o futuro, problemas e desafios a serem superados continuam principalmente em termos da assistência médica para os indivíduos já infectados e da manutenção da vigilância epidemiológica ${ }^{5-6}$.

A soroprevalência da população rural do país, determinada por inquérito sorológico realizado entre 1975 e 1980, por amostragem em todos os municípios (à exceção daqueles do Estado de São Paulo, onde as atividades de controle estavam em curso desde meados dos anos 60 ), era de $4,2 \%$ para a população geral rural brasileira, com maiores taxa de prevalência em Minas Gerais e Rio Grande do Sul $(8,8 \%)^{7}$.

De acordo com Dias ${ }^{5}$, a prevalência da doença de Chagas era 4,7\% na década de 1970; porém, em recentes estudos caiu para menos de $0,2 \%{ }^{8}$. O presente estudo vem mostrar a situação atual da doença de Chagas em uma área de Minas Gerais. Apesar da pobreza de algumas regiões mineiras, a doença tem sido controlada.

Em inquérito sorológico realizado no Vale de Jequitinhonha para verificar a situação atual da transmissão desta endemia, foram selecionadas 1.412 crianças entre 7 a 14 anos, demonstrando uma soroprevalência de $0,4 \%{ }^{9}$. Dias e cols ${ }^{10}$ concluíram que em Lassance, município da descoberta de Carlos Chagas, entre 1908 e 2001, através de registros históricos e pesquisas atuais, a infecção chagásica é residual, com prevalência geral de $5 \%$, afetando basicamente os grupos etários elevados, não se encontrando soropositivos abaixo de 20 anos de idade. 
O perfil clínico-epidemiológico dos chagásicos detectados é o habitual com área de transmissão interrompida, com a maioria dos casos na forma cardíaca benigna ou crônica indeterminada. A prevalência da doença de Chagas em Serra Azul foi de 2,1\%, com chagásicos adultos. Os achados do presente estudo são semelhantes em alguns aspectos ao panorama atual da doença em Lassance, ou seja, um resíduo de indivíduos mais idosos portadores da doença de Chagas, com a qual se infectaram há duas décadas ou mais, a merecer atenção médica e social. São pessoas de origem rural (93\%), geralmente pobres e de baixa escolaridade, sendo a transmissão vetorial a mais provável fonte de infecção, como de regra se observa nos municípios mineiros ${ }^{11}$. De fato, no nosso estudo a idade média dos soropositivos foi de 67 anos, não se evidenciando nenhuma criança ou adulto jovem com exame sorológico positivo.

A falta de investigações sobre a doença de Chagas em indivíduos idosos contrasta com a importância da enfermidade nessa faixa etária para a compreensão de muitos pontos ainda obscuros. $\mathrm{O}$ aumento da média de idade dos indivíduos com doença de Chagas pôde ser esperado com a interrupção de sua transmissão, o que significa um grande desafio para os médicos e para o sistema de saúde nas próximas décadas 5 .

Observando-se as co-morbidades encontradas em estudo prévio, as doenças circulatórias apresentavam a prevalência de $33 \%$ (hipertensão arterial sistêmica e doenças isquêmicas coronarianas) ${ }^{12}$. No presente estudo, houve elevada prevalência de hipertensão arterial (64\%), provavelmente pela faixa etária mais alta da população sorologicamente positiva.

O Consenso Brasileiro sobre a Doença de Chagas ${ }^{13}$ define, para fins operacionais, a cardiopatia chagásica crônica pela presença de anormalidades eletrocardiográficas sugestivas de comprometimento cardíaco, em indivíduo sintomático ou não. No presente estudo, baseando-se nos achados de exame físico e eletrocardiograma, sete indivíduos (50\%) foram considerados portadores de cardiopatia chagásica, definida pela presença de distúrbio avançado de condução do ramo direito, alteração eletrocardiográfica típica da doença de Chagas. A insuficiência cardíaca estava presente em dois pacientes com apresentação clínica da cardiopatia chagásica, sendo que um evolui para o óbito. Nossos achados estão semelhantes aos da literatura, verificando-se que em torno de $10 \%$ dos indivíduos apresentam formas graves da cardiopatia chagásica ${ }^{5,14}$.

Os achados eletrocardiográficos no presente estudo estão de acordo com os dados da literatura. Rosembaum e Alvarez $z^{15}$ descreveram 113 pacientes chagásicos e $86,9 \%$ dos eletrocardiogramas com anormalidades, com incidência de bloqueio do ramo direito em $55,7 \%$ dos soropositivos ${ }^{15}$. Em nosso estudo, foi encontrado bloqueio de ramo direito em metade dos indivíduos com sorologia positiva, diferente dos controles. A segunda alteração mais encontrada foi a extrassístole ventricular, detectada em cinco (36\%) indivíduos. Este achado, de acordo com a literatura, pode variar de 6 a $55 \%$ dos indivíduos soropositivos ${ }^{10}$. A alta porcentagem de alterações eletrocardiográficas nos soropositivos sugere forte dependência da etiologia chagásica. As anormalidades eletrocardiográficas presentes nas outras doenças associadas, como hipertensão arterial, diabetes mellitus e insuficiência coronariana, seriam alterações da repolarização ventricular, hipertrofia ventricular e inatividade elétrica, que não representam achados característicos na doença de Chagas.
Em conclusão, a prevalência da doença de Chagas no povoado de Serra Azul, centro-oeste de Minas Gerais foi de 2,1\%, com pessoas soropositivos pertencendo aos grupos etários mais elevados, sugerindo controle da transmissão nesta área endêmica. A população estudada reside principalmente em área rural, com baixo nível de escolaridade e em condições de subemprego. As anormalidades eletrocardiográficas foram frequentes, com predomínio do distúrbio de condução do ramo direito.

\section{CONFLITO DE INTERESSE}

Os autores declaram não haver nenhum tipo de conflito de interesse no desenvolvimento do estudo.

\section{REFERÊNCIAS}

1. Word Health Organization. Chagas'disease, Brazil. Wkly Epidemiol Rec 2000; 75:153-155

2. Word Health Organization. Expert Commitee on the Control of Chagas'disease. Control of Chagas disease: Second Report no the WHO Expert Committee. Geneva, 2002.

3. Word Health Organization. Unicef-Undp. Word Bank. Special Programme for Research and Trining in Tropical Disease. Chagas' disease. Disponível em http:/ / who.int/tdr/dw/chagas2003.htm.

4. Dias JCP. Chagas disease: success and challenges. Cad Saúde Pública 2006; 22: 2020-2021.

5. Dias JCP. Globalização, iniqüidade e doença de Chagas. Cad Saúde Pública 2007; 11:13-22.

6. Dias JC. Epidemiological surveillance of Chagas disease. Cad Saúde Pública 2000; 16: 43-59.

7. Ministério da Saúde. Consenso Brasileiro em Doença de Chagas. Diagnóstico e manejo clínico da forma cardíaca da doença de Chagas. Rev Soc Bras Med Trop $2005 ; 38: 15-18$.

8. Silveira AC. Situação do controle da transmissão vetorial da doença de Chagas nas Américas. Cad Saúde Pública 2000;16:35-42.

9. Borges JD, Assis GFM, Gomes LV, Dias JCP, Pinto IDM, Martins-Filho AO, et al Seroprevalence of Chagas disease in schoolchildren from two municipalities of Jequitinhonha Valley, Minas Gerais, Brazil; six years following the onset of epidemiological surveillance. Rev Inst Med Trop São Paulo 2006; 48:81-86.

10. Dias JCP, Machado EMM, Borges EC, Moreira EF, Gontijo C, Azeredo BVM Doença de Chagas em Lassance, MG Reavaliação clínico epidemiológica 90 anos após a descoberta de Carlos Chagas. Rev Soc Bras Med Trop 2002; 35:167 176.

11. Dias JCP. Doença de Chagas em Bambuí, Minas Gerais, Brasil. Estudo Cínicoepidemiológico a partir da fase aguda entre 1940 e 1982. Universidade Federal de Minas Gerais, Belo Horizonte. 1982.

12. Carvalho ME, Silva RA, Barata JM, Domingos MF, Ciaravolo RM, Zacharias F. Chagas' disease in the southern coastal region of Brazil. Rev Saúde Pública 2003; 37: 49-58.

13. Ministério da Saúde. Consenso Brasileiro em Doença de Chagas. Diagnóstico e manejo clínico da forma cardíaca da doença de Chagas. Rev Soc Bras Med Trop 2005 ; 38:15-18.

14. Chagas C, Villela E. Forma Cardíaca da Trypanosomíase Americana. Mem Inst Osvaldo Cruz 1922; 14:5-61.

15. Rosembaum MB, Alvarez AJ. The eletrocardiogram in chronic chagasic myocarditis. Am Heart J 1955; 50:492-527.

16. Ribeiro ALP. Eletrocardiografia Ambulatorial na Doença de Chagas. Rev Soc Bras Med Trop 1994; 27:55-56. 